\title{
Mechanical intestinal obstruction due to phytobezoar: Retrospective cohort study
}

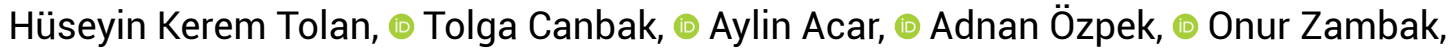

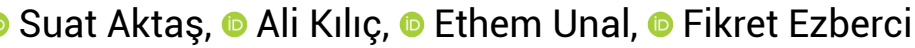 \\ Department of General Surgery, Health Sciences University, Umraniye Training and Research Hospital, Istanbul, Turkey
}

\begin{abstract}
Introduction: In this study, we aimed to look through our patients with intestinal obstruction (IO) due to phytobezoars.

Materials and Methods: Patients with intestinal obstruction due to phytobezoar between April 2013 and January 2017 were evaluated in this study.

Results: There were 13 patients. Seven patients were female; six patients were male, and the mean age was 54.6. Six of the patients had a history of gastric re-section, two patients had severe tooth disturbances, and two patients had diabetes. In four patients, there was a story of eating persimmon (diospyrobezoar). Three of the patients were diagnosed with computerized tomography (CT) and nine of the patients with the laparotomy. Two patients had bezoars in the endoscopy. Two patients were treated nonoperatively. Intravenous (IV) fluid, nasogastric decompression, oral cola beverage and IV metoclopramide were administered. The patients requiring surgery were operated within 72 hours. In the laparotomy, bezoars were found in the ileum and four also in the stomach. In three of the patients, bezoars were crushed manually and then transferred to ileum; in eight of the patients, bezoars were extracted with enterotomy. Two of the patients who had enterotomy, underwent re-laparotomy due to a leak, and these two patients were lost. Superficial wound infection developed in four patients. Specimens were reported as a phytobezoar in pathology reports.

Conclusion: Phytobezoars should be kept in mind in differential diagnosis in ileus patients with a gastric operation history and dental problems. Patients' detailed anamnesis and eating habits should be questioned. CT and endoscopy may help. Patients with partial obstruction may benefit from conservative treatment.

Keywords: Emergency; obstruction; phytobezoar.
\end{abstract}

\section{Introduction}

Bezoar is the hardened form of eaten materials. Bezoars are massive formations caused by indigestible foods or other substances like medicine, hair in the gastrointestinal tract. They are called phytobezoars when they are with agents such as vegetable and fruit fibers. Phytobezoars are indigestible materials composed of vegetable-derived cellulose fibers and can be found in the entire gastrointestinal tract thus may cause an intestinal obstruction. There are different types of bezoars named due to their 
contents. ${ }^{[1]}$ Clinical presentation of a bezoar-induced small bowel obstruction with an acute surgical abdomen is very rare, occurring in $1.1 \%$ of the case. ${ }^{[2]}$ We aimed to evaluate the patients who presented to the emergency department with mechanical intestinal obstruction due to phytobezoar and discuss with the literature.

\section{Materials and Methods}

Patients with mechanical intestinal obstruction (MIO) due to phytobezoar between April 2013 and January 2017 were evaluated. The study was carried out in accordance with the Helsinki Declaration of Principles. Informed consent form was taken from all patients included in the study. Patients were classified according to their age, gender, history of previous operation, nutrition style, diagnostic methods, treatment used, morbidity and mortality. Different treatment modalities were performed. Some had the passage of bezoar and relief of symptoms but some had even more than one surgery that resulted with mortality. All statistical analyses were performed using SPSS 22.0 statistical package software (SPSS, Inc., Chicago, IL, USA). Categorical variables were expressed as frequencies and percentages.

\section{Results}

There were 13 patients who had MIO due to bezoar. Seven (53.8\%) were female, 6 (46.2\%) were male and mean age was 54.6 years (range 32-72). Six of the patients had history of gastric resection, 2 had severe tooth disturbances and 2 had diabetes. In four patients there was a story of eating persimmon (diospyrobezoar). Three of the patients were diagnosed with computerized tomography (CT) and 9 with the laparotomy. Two patients had gastric bezoars in the upper GI endoscopy.

Two patients (15.4\%) were treated non-operatively. IV fluid replacement, nasogastric decompression, oral cola beverage and IV metoclopramide were administered. The patients requiring surgery were operated within 72 hours of admission. In the laparotomy; bezoars were found in the ileum and in 4 also there was a bezoar also in the stomach. In $3(27.7 \%)$ of the patients, bezoars were crushed manually and then transferred to ileum; in 8 (72.3\%), bezoars were extracted with enterotomy (Fig. 1). Two of the patients (25\%) who had enterotomy, underwent re-laparatomy due to a leak and these 2 patients died due to the complications of the gastrointestinal leak. Superficial wound infection developed in four patients (36.4\%). Specimens were reported as a phytobezoar in the final pathology reports Table 1.

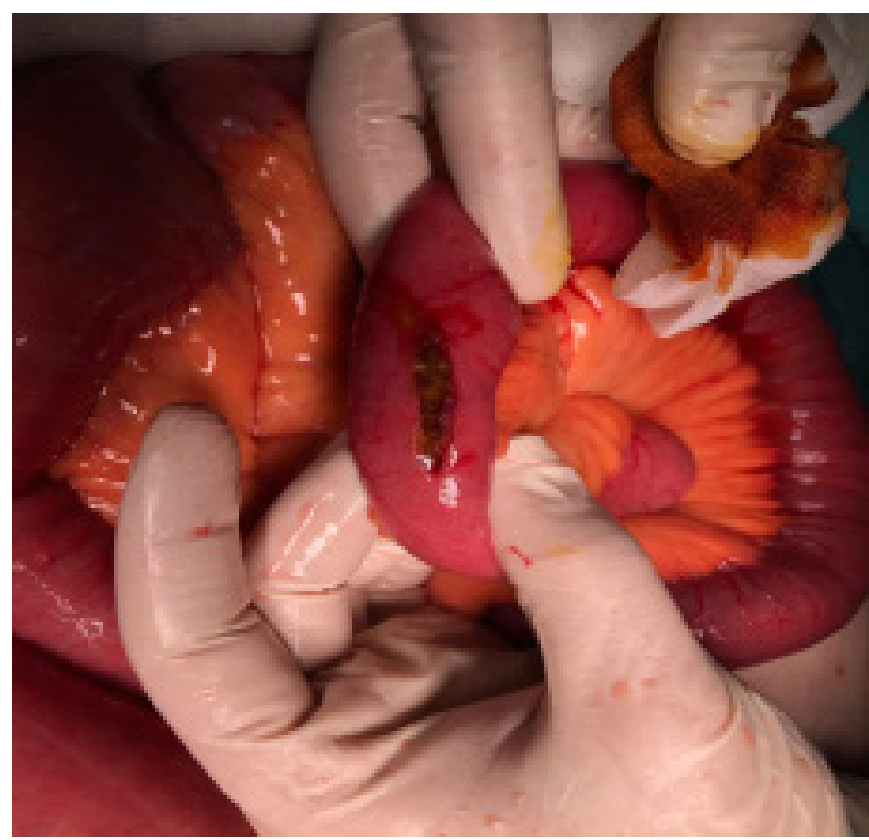

Figure 1. Treatment with enterotomy.

\section{Discussion}

Small bowel obstruction is a common acute presentation in any general surgical unit. However, its preoperative diagnosis and management may often be difficult because of its myriad causes. Unlike post-operative adhesions; which accounts for $60-80 \%$ of all cause, small bowel obstruction secondary to bezoar impaction is considerably less common, with the reported frequency around 0.4$4 \%{ }^{[3,4]}$ Phytobezoars, trichobezoars, pharmacobezoars, lactobezoars, persimmon phytobezoar (diospyrobezoar) are 5 most common types of bezoars. The most common risk factors for phytobezoar formation are previous gastric surgery, poor mastication, overindulgence of food with high fiber content, diabetes mellitus complicated by gastroparesis and coexisting diseases with delayed gastric emptying. ${ }^{[5]}$ The most common type is phytobezoars, which are composed of undigested fiber from vegetables or fruits especially persimmons. ${ }^{[6]}$

Mihai et al. ${ }^{[7]}$ noted that there were 49 cases of gastric bezoars over a period of 20 years $(0.068 \%$ of all endoscopies). Clinical presentation of a bezoar-induced SBO with an acute surgical abdomen is very rare, occurring in $1.1 \%$ of the case. ${ }^{[2]}$ They usually form in the stomach, but they can migrate in to the small bowel, where they may cause an obstruction. ${ }^{[8]}$

Depending on the location and size, clinical manifestations of gastrointestinal bezoars can vary such as abdominal pain, bloody or tarry stool, abdominal fullness, 
anemia-or even upper GI bleeding-or signs of intestinal obstruction due to large intestinal bezoars. ${ }^{[9]}$

The most common symptom of bezoar-induced SBO is abdominal pain (96-100\%). Other common symptoms include nausea and vomiting. ${ }^{[4]}$ In our patients the signs and symptoms were similar with the literature.

They usually are impacted in the narrowest part of the intestine especially in the terminal ileum and ileocecal valve. Surgical options reported are manual fragmentation of bezoar and pushing it toward cecum. In $3(27.7 \%)$ of our patients the bezoars were successfully crushed manually and then they were transferred in to the distal part of the bowel. If bezoars cannot be removed by milking then an enterotomy should be done to remove the bezoar. Segmental bowel resection and anastomosis may also be required. ${ }^{[2,4]}$ In our series in $8(72.3 \%)$ of the patients, bezoars were extracted with an enterotomy. Two of the patients (25\%) who had an enterotomy, underwent re-laparatomy due to a leak and these 2 patients unfortunately died due to the complications of the gastrointestinal leak from the anastomosis.

The patients that had a bariatric surgery; are also more prone to bezoar formation due to their potential eating disorders and because of the gastro-enterostomy made on to a small gastric pouch after the Roux-en-Y gastric bypass surgery. Possibility of a bezoar formation should be kept in mind in Roux-en-Y gastric bypass patients who has nausea and vomiting complaints. ${ }^{[10]}$

Spontaneous disappearance of a bezoar under the absence of specific treatment was also observed in some patients. ${ }^{[11]}$ Likewise in our series we had two patients $(15.4 \%)$ that were treated non-operatively only with the conservative treatment. IV fluid replacement, nasogastric decompression, oral cola beverage and IV metoclopramide were administered and during their follow-ups the ileus was resolved without needing any extra interventions.

All the patients had postoperative nutritional counseling in regard to prevent the recurrence of a bezoar formation. Guidelines include proper chewing of food, plenty of liquids with meals to drink coke time to time which helps in chemical degradation of the bezoars and avoidance of a high fiber diet. ${ }^{[12,13]}$

In conclusion, phytobezoars are a rare cause of mechanical intestinal obstruction. It should be kept in mind in differential diagnosis in MIO patients with a gastric operation history and dental problems. Patients' detailed anamnesis and eating habits should be questioned. CT and endoscopy may help but not always. It should be suspected in patients with potential predisposing factors. Patients with partial obstruction may benefit from conservative treatment. Different surgical methods can be used depending on the; location, mechanical degradability and adhesiveness of the bezoar.

Table 1. Datas of cases

\begin{tabular}{lccccc} 
Patients & Age & Gender & Etiology/History & Diagnosis with & Treatment \\
\hline 1 & 32 & Female & Diospyrobezoar & CT & Surgery \\
2 & 48 & Male & Diospyrobezoar & Laparotomy & Surgery \\
3 & 56 & Male & Severe tooth disturbances & Laparotomy & Surgery \\
4 & 67 & Female & History of gastric resection & Endoscopy & Medical \\
5 & 58 & Female & Diabetes mellitus & Laparotomy & Surgery \\
6 & 45 & Male & Diospyrobezoar & CT & Surgery \\
7 & 54 & Female & Severe tooth disturbances & Laparotomy & Surgery \\
8 & 64 & Male & History of gastric resection & Laparotomy & Surgery \\
9 & 59 & Male & History of gastric resection & Endoscopy & Medical \\
10 & 72 & Female & History of gastric resection & Laparotomy & Surgery \\
11 & 57 & Female & Diabetes mellitus & CT & Surgery \\
12 & 35 & Male & Diospyrobezoar & Laparotomy & Surgery \\
13 & 63 & Female & History of gastric resection & Laparotomy & Surgery \\
\hline
\end{tabular}




\section{Disclosures}

Ethichs Committee Approval: The ethical committee approval was not needed due to the retrospective design of the study.

Peer-review: Externally peer-reviewed.

Conflict of Interest: None declared.

Authorship Contributions: Concept - H.K.T., T.C., A.A.; Design - H.K.T., A.K., A.Ö.; Supervision - E.Ü., F.E.; Materials - H.K.T., O.Z., A.Ö.; Data collection and/or processing -HKT, AK, AA.; Analysis and/or interpretation T.C., A.A.; Literature search - S.A., H.K.T., O.Z.; Writing - H.K.T., A.A.; Critical review - F.E.

\section{References}

1. Sanders MK. Bezoars: from mystical charms to medical and nutritional management. Pract Gastroenterol 2004;18:3750.

2. Bedioui H, Daghfous A, Ayadi M. A report of 15 cases of small bowel obstruction secondary to phytobezoars: predisposing factors and diagnostic difficulties. Gastroenterol Clin Biol 2008;32:596-600. [CrossRef]

3. Salemis N, Panagiotopoulos N, Sdoukos N, Niakas E. Acute surgical abdomen due to phytobezoar-induced ileal obstruction. J Emerg Med 2013;44:21-3. [CrossRef]

4. Erzurumlu K, Malazgirt Z, Bektas A, Dervisoglu A, Polat C, Senyurek G. Gastrointestinal bezoars: a retrospective anal- ysis of 34 cases. World J. Gastroenterol 2005;11:1813-7.

5. Lee J. Bezoars and foreign bodies of the stomach. Gastrointest Endosc Clin N Am 1996;6:605-19. [CrossRef]

6. Sarhan M, Shyamali B, Fakulujo A, Ahmed L. Jejunal bezoar causing obstruction after laparoscopic Roux-en-Y gastric bypass. JSLS 2010;14:592-5. [CrossRef]

7. Mihai C, Mihai B, Drug V, Cijevschi Prelipcean C. Gastric bezoars-diagnostic and therapeutic challenges. J Gastrointestin Liver Dis 2013;22:111.

8. Escamilla C, Robles-Campos R, Parrilla-Paricio P, LujanMompean J, Liron-Ruiz R, Torralba-Martinez JA. Intestinal obstruction and bezoars. J. Am. Coll. Surg 1994;179:285-8.

9. Baongoc Nasri, Marius Calin, Ajay Shah, Brian Gilchrist. A rare cause of small bowel obstruction due to bezoar in a virgin abdomen. Int J Surg Case Rep 2016;19:144-6. [CrossRef]

10. Ertugrul I, Tardum Tardu A, Tolan K, Kayaalp C, Karagul S, Kirmizi S. Gastric bezoar after Roux-en-Y gastric bypass for morbid obesity: A case report. Int J Surg Case Rep 2016;23:112-5. [CrossRef]

11. Iwamuro M, Tanaka S, Shiode J, Imagawa A, Mizuno M, Fujiki $S$, et al. Clinical characteristics and treatment outcomes of nineteen Japanese patients with gastrointestinal bezoars. Intern Med 2014;53:1099-105. [CrossRef]

12. Parsi S, Rivera C, Vargas J, Silberstein M. Laparoscopicassisted extirpation of a phytobezoar causing small bowel obstruction after Roux-en-Y laparoscopic gastric bypass. Am Surg 2013;79:93-5. [CrossRef]

13. Ladas SD, Triantafyllou K, Tzathas C. Gastric phytobezoars may be treated by nasogastric coca cola lavage. Eur. J. Gastroenterol. Hepatol 2002;14:801-3. [CrossRef] 